\title{
How to predict and achieve success with a very long procedure in an endoscopy unit: Is it time for a break?
}

\section{(6)(1) $\odot$}

\author{
Author \\ Hubert Piessevaux \\ Institution \\ Department of Hepatogastroenterology, Cliniques \\ universitaires Saint-Luc - Université catholique de Louvain, \\ Brussels, Belgium \\ Bibliography \\ DOI https://doi.org/10.1055/a-0889-7945 | \\ Endoscopy International Open 2019; 07: E1097-E1098
}

\author{
(c) Georg Thieme Verlag KG Stuttgart · New York \\ eISSN 2196-9736
}

Corresponding author

Hubert Piessevaux, Cliniques universitaires Saint-Luc, Dept

of Hepatogastroenterology, Avenue Hippocrate 10 ,

Bruxelles 1200, Belgium

Fax: + 3227648927

hubert.piessevaux@uclouvain.be
Most advanced therapeutic procedures currently performed in modern endoscopy units substantially gained in efficiency compared to a not-so-distant past. The advent of therapeutic channel endoscopic ultrasound scopes, efficient endoscopic retrograde cholangiopancreatography guidewires, over-the-scope clips, self-expanding metal stents, lumen-apposing metal stents, and numerous other technical improvements helped to shorten the duration of sometimes very complex procedures. One notable exception to this evolution is colorectal endoscopic submucosal dissection (ESD). The reason for this exception is related to absence of a technical size limit for tumors that can be removed using this technique. The European Society of Gastrointestinal Endoscopy guidelines, for example, recommend use of ESD for all lesions larger than $20 \mathrm{~mm}$ with high likelihood of malignancy but do not report an upper limit of size [1]. In the rectum, more specifically, very large lesions which often extend to the dentate line would require mutilating surgery with poor functional outcome, thereby justifying an organ-sparing ESD approach if possible.

Theoretically, two factors will determine duration of a colorectal ESD procedure: the area of the lesion and the dissection speed. In many colorectal ESD series, lesions larger than $100 \mathrm{X}$ $100 \mathrm{~mm}$ (i.e. $78.5 \mathrm{~cm}^{2}$ assuming a circular shape) are not uncommon [2]. At a pace of $10 \mathrm{~cm}^{2}$ per hour reported in experienced European series, this would predict a procedure time of about 8 hours [3]! A recent very interesting study by lacopini et al. studied predictors of difficult dissection in colorectal ESD and defined a scoring system based on preoperative findings enabling anticipation of dissection difficulties [4]. This score includes operator experience, presence of a scar, laterally spreading tumor (LST) type and sessile presentation as predictors. We have shown in our experience that non-lifting, LST-non-granu- lar morphology and difficult retroflexed approach were independent risk factors associated with the need for conversion to rescue endoscopic mucosal resection, while tumor size and location were not [5]. We thus currently have tools to anticipate a procedure that will last for a longer period of time.

How can one cope with such long procedures? Excessive procedure duration may both have an impact on the patient outcome and safety but also on the mental or physical wellbeing of the endoscopist. Very little is known on these subjects in gastrointestinal endoscopy, but some data are available in the surgical literature indicating a negative impact on the operator's wellbeing [6]. Ergonomic factors, but also experience, handedness or height may play a role in the outcome for the provider. Moreover, and this finding can potentially be extrapolated to gastrointestinal endoscopy, a lack of direct visual or physical access to target anatomy, reduction in degrees of freedom, the fulcrum effect, and poorly designed tools, all create awkward work postures, and a limiting two-dimensional view of the operative field, may generate physical stress [7]. Interestingly, some beneficial interventions have been suggested, such as intraoperative micro breaks, which may not only improve physical symptoms, but also the operator's mental status [8]. Finally, the organizational implications of very long procedure durations in an endoscopy unit are not to be underestimated.

In this issue of EIO, Kawaguti et al. report on a new approach to tackle this problem. To preserve the advantage of en-bloc resection of very large rectal lesions while simultaneously alleviating the burden of very long procedure times, they decided to perform a two-step ESD. In this paper, the authors report their experience with four patients in which the rectal ESD procedure for very large lesions was interrupted halfway and re- 
sumed 2 days later. Implementing such a strategy requires that technical resumption be possible with only limited additional difficulties, that there are no added safety issues, and that specimen quality would not be degraded. All three requirements were somehow met. The authors did succeed in en-bloc resection in all cases with an average total duration procedure around 8 hours. There were no safety issues. Importantly, while the pathologist reported an area of ischemic damage to the specimen, global evaluation of the resected tumor seemed not to be affected.

Can this approach readily be recommended in lesions to be resected using ESD in which lesion size and predictors suggest a very long procedure duration? A few words of caution are still necessary. First, the number of cases is still too low to advocate the safety of this approach. Secondly, the experience of Kawaguti et al is limited to the rectum and there is no assurance that their findings can be extrapolated to the colon. Thirdly, the authors report on benign cases. The ultimate advantage of colorectal ESD over large-field piecemeal EMR resides in treatment of patients with shallow submucosal invasion without other deleterious predictive factors such as lymphovascular invasion, poor tumor differentiation or tumor budding. It remains to be validated that these very important prognostic factors can correctly be evaluated in the ischemic areas of the specimen [9]. Finally, the proposed approach comes with the additional costs of an extended hospital stay, a second general anesthesia, and a second set of consumables such as the dissection knife and hemostatic forceps.

In summary, very long procedural durations are an issue, more specifically in colorectal ESD. They may have an impact not only on the patient but also on the operator's wellbeing. We have some predictor tools to anticipate this situation. In patients with very large rectal LSTs with low risk of submucosal infiltration, a two-step approach may be valuable.
Competing interests

None

References

[1] Pimentel-Nunes P, Dinis-Ribeiro $M$ et al. Endoscopic submucosal dissection: European Society of Gastrointestinal Endoscopy (ESGE) Guideline. Endoscopy 2015; 47: 829-854

[2] Akintoye E, Kumar N, Aihara H et al. Colorectal endoscopic submucosal dissection: a systematic review and meta-analysis. Endosc Int Open 2016; 4: E1030-E1044

[3] Rönnow CF, Uedo N, Toth E et al. Endoscopic submucosal dissection of 301 large colorectal neoplasias: outcome and learning curve from a specialized center in Europe. Endosc Int Open 2018; 6: E1340 -E1348

[4] Iacopini F, Saito Y, Bella A et al. Colorectal endoscopic submucosal dissection: predictors and neoplasm-related gradients of difficulty. Endosc Int Open 2017; 5: E839-E846

[5] Pérez-Cuadrado-Robles E, Snauwaert C, Moreels TG et al. Risk factors for conversion to snare resection during colorectal endoscopic submucosal dissection in an expert Western center. Endoscopy 2019; 51 : $152-160$

[6] Thiels CA, Lal TM, Nienow JM et al. Surgical never events and contributing human factors. Surgery 2015; 158: 515- 521

[7] Park A, Lee G, Seagull F] et al. Patients benefit while surgeons suffer: an impending epidemic. J Am Coll Surg 2010; 210: 306-313

[8] Park AE, Zahiri HR, Hallbeck MS et al. Intraoperative "micro breaks" with targeted stretching enhance surgeon physical function and mental focus: a multicenter cohort study. Ann Surg 2017; 265: 340 346

[9] Dessain A, Snauwaert C, Baldin P et al. Endoscopic submucosal dissection specimens in early colorectal cancer: lateral margins, macroscopic techniques, and possible pitfalls. Virchows Arch 2017; 470: $165-174$ 